\title{
A comparative study of dose distribution of PBT, 3D-CRT and IMRT for pediatric brain tumors
}

\author{
Daichi Takizawa 1* Masashi Mizumoto ${ }^{1}$, Tetsuya Yamamoto ${ }^{2}$, Yoshiko Oshiro ${ }^{1}$, Hiroko Fukushima ${ }^{3}$, \\ Takashi Fukushima ${ }^{3}$, Toshiyuki Terunuma ${ }^{4}$, Toshiyuki Okumura ${ }^{1}$, Koji Tsuboi ${ }^{1}$ and Hideyuki Sakurai ${ }^{1}$
}

\begin{abstract}
Introduction: It was reported that proton beam therapy (PBT) reduced the normal brain dose compared with X-ray therapy for pediatric brain tumors. We considered whether there was not the condition that PBT was more disadvantageous than intensity modulated photon radiotherapy (IMRT) and 3D conventional radiotherapy (3D-CRT) for treatment of pediatric brain tumors about the dose reduction for the normal brain when the tumor location or tumor size were different.

Methods: The subjects were 12 patients treated with PBT at our institute, including 6 cases of ependymoma treated by local irradiation and 6 cases of germinoma treated by irradiation of all four cerebral ventricles. IMRT and 3D-CRT treatment plans were made for these 12 cases, with optimization using the same planning conditions as those for PBT. Model cases were also compared using sphere targets with different diameters or locations in the brain, and the normal brain doses with PBT, IMRT and 3D-CRT were compared using the same planning conditions.

Results: PBT significantly reduced the average dose to normal brain tissue compared to 3D-CRT and IMRT in all cases. There was no difference between 3D-CRT and IMRT. The average normal brain doses for PBT, 3D-CRT, and IMRT were 5.1-34.8\% (median 14.9\%), 11.0-48.5\% (23.8\%), and 11.5-53.1\% (23.5\%), respectively, in ependymoma cases; and $42.3-61.2 \%$ (48.9\%), 54.5-74.0\% (62.8\%), and 56.3-72.1\% (61.2\%), respectively, in germinoma cases. In the model cases, PBT significantly reduced the average normal brain dose for larger tumors and for tumors located at the periphery of the brain.
\end{abstract}

Conclusion: PBT reduces the average dose to normal brain tissue, compared with 3D-CRT and IMRT. The effect is higher for a tumor that is larger or located laterally.

Keywords: Brain, 3D-CRT, IMRT, PBT, Pediatric tumor

\section{Introduction}

Deterioration of intelligence after radiotherapy is an important problem in growing children. The degree of deterioration is affected by the irradiation dose, volume, site, and age at irradiation [1]. A major concern of brain irradiation in pediatric patients is subsequent deterioration of intelligence [6-10], since neurodevelopment is affected by the treatment dose and age at irradiation $[1,11,12]$. In 4 patients among 27 children with medulloblastoma treated

\footnotetext{
* Correspondence: dtakizawa@pmrc.tsukuba.ac.jp

${ }^{1}$ Department of Radiation Oncology, University of Tsukuba, Tsukuba, Ibaraki, Japan

Full list of author information is available at the end of the article
}

with craniospinal irradiation with a posterior fossa boost, Walter et al. [8] found an IQ decline of 3.9 points per year during a median observation period of 4.8 years, and cognitive losses did not seem to have reached a plateau. Merchant et al. analysed the correlation with the degree of deterioration and DVH of the whole brain and suggested that IQ can be obtained from the following formula [1]:

$$
\begin{aligned}
\mathrm{IQ}= & 93.11 \pm(0.028 \times \text { age }-0.0095 \\
& \times \text { average dose to the brain }) \times \text { time }
\end{aligned}
$$

Intensity modulated photon radiotherapy (IMRT) is now widely used and can reduce the dose to an at-risk organ and decrease the high dose area, but the low dose 
area is wider than 3D-CRT. [13] X. Sharon et al. found that IMRT redused temporal lobe dose compared with 3D-CRT [14], but it is possible that the dose reduction for temporal lobe by IMRT increase the dose of the other lobes, and don't reduce average dose to the brain compare with 3DCRT. In contrast, proton beam therapy (PBT) has a sharp energy peak, referred to as the Bragg peak, which produces excellent dose localization and reduces the dose to normal tissue $[2,15,16]$. Therefore, excellent tumor coverage can be achieved with a small number of beam ports, and several reports have shown advantages of PBT compared to photon radiotherapy, including IMRT. [3] The advantages of PBT compared to photon radiotherapy including IMRT have been described in treatment of pediatric CNS tumors [9-11]. MacDonald et al. found that proton beams can achieve tumor coverage as well as IMRT, but that normal tissue sparing was better in PBT for patients with germ cell tumor and ependymoma $[17,18]$.

We conduct PBT for all pediatric patients with brain malignancies who are indicated for photon radiotherapy in order to reduce the normal brain dose $[4,5]$. However, the conditions under which the advantages of PBT are maximized are unclear; for example, a large or small tumor, local or whole-ventricle irradiation, and a peripheral or central tumor. In this study, we evaluated the more advantageous condition of PBT for pediatric brain tumors in comparison to 3D-CRT and IMRT, using quantitative analysis of localized irradiation for ependymoma cases and wholeventricular irradiation for germinoma cases, and model cases that targets were different size or location.

\section{Patients and methods}

\section{Patients}

The subjects were 12 pediatric patients (Table 1) with brain tumors treated with PBT at our institute from 2009 to 2011 . The study was approved by the institutional review board at our institution. Six patients (3 males, 3 females; median age 4 [range: 2-6] years old) had ependymoma and 6 had germinoma ( 3 males, 3 females; median age 13 [range: 10-16] years old). Localized and whole-ventricle irradiation was performed for ependymoma and germinoma, respectively, during which the patients were immobilized using individually manufactured thermoplastic masks.

\section{Comparison of PBT, IMRT, and 3D-CRT}

Proton beams from 155 to $250 \mathrm{MeV}$, generated through a linear accelerator and synchrotron, were spread out and shaped with ridge filters, double-scattering sheets, multicollimators, and custom-made boluses to ensure that the beams conformed to the treatment planning data. Planning CT images were taken at $2-\mathrm{mm}$ intervals. IMRT and 3D-CRT treatment plans were generated and optimized using the same practical treatment planning $\mathrm{CT}$ as that used for PBT to compare dose distributions among the three methods. The prescribed doses were the same in each cases among the three methods, 45Gy to 61.2Gy (median 52.2Gy) in ependymoma cases as local irradiation, and 24Gy to 30.6Gy (median 30.6Gy) in germinoma cases as whole ventricle irradiation. All PBT plans were prescribed the same dose with IMRT/3DCRT as the equivalent dose. The clinical target volume (CTV) for ependymoma was defined as the surgical defect plus a margin of 0.5 to $1 \mathrm{~cm}$. The CTV for germinoma was defined as all cerebral ventricles. The same planning target volume (PTV) and at-risk organ was re-contoured for the photon radiotherapy plans. The PTV was identical for PBT, IMRT, and 3D-CRT for each patient and was defined by the $95 \%$ iso-dose line in all plans. The maximal dose prescriptions for at-risk organs were $<4$ Gy for the lens and $<50$ Gy for the brainstem and chiasma. We used helical tomotherapy that delivered 51

Table 1 Clinical background of patients

\begin{tabular}{|c|c|c|c|c|c|c|c|c|c|}
\hline No. & Age (y) & Sex & Tumor & CTV1 (cC) & $\begin{array}{l}\text { Dose to normal } \\
\text { brain (\%) [3D-CRT] }\end{array}$ & $\begin{array}{l}\text { Dose to normal } \\
\text { brain (\%) [IMRT] }\end{array}$ & $\begin{array}{l}\text { Dose to normal } \\
\text { brain (\%) }[\mathrm{PBT}]\end{array}$ & $\begin{array}{l}\text { The relative decrease (\%) } \\
\text { (3DCRT-PBT)/3DCRT }\end{array}$ & $\begin{array}{l}\text { The relative decrease } \\
\text { (\%) (IMRT-PBT)/IMRT }\end{array}$ \\
\hline 1 & 6 & M & Ependymoma & 6.9 & 11.0 & 11.5 & 5.1 & 53.6 & 55.7 \\
\hline 2 & 6 & M & Ependymoma & 74.0 & 20.5 & 20.1 & 14.4 & 29.8 & 28.4 \\
\hline 3 & 2 & $\mathrm{~F}$ & Ependymoma & 6.1 & 17.8 & 17.1 & 6.5 & 63.5 & 62.0 \\
\hline 4 & 3 & $\mathrm{~F}$ & Ependymoma & 42.5 & 30.0 & 31.8 & 15.4 & 48.7 & 51.6 \\
\hline 5 & 4 & M & Ependymoma & 116.3 & 48.5 & 53.1 & 34.8 & 28.2 & 34.5 \\
\hline 6 & 3 & $\mathrm{~F}$ & Ependymoma & 48.0 & 27.0 & 26.9 & 16.7 & 38.1 & 37.9 \\
\hline 7 & 11 & M & Germinoma & 344.8 & 74.0 & 72.1 & 61.2 & 17.3 & 15.1 \\
\hline 8 & 16 & $\mathrm{~F}$ & Germinoma & 150.2 & 60.5 & 61.8 & 48.5 & 19.8 & 21.5 \\
\hline 9 & 10 & M & Germinoma & 168.8 & 54.5 & 56.3 & 42.3 & 22.4 & 24.9 \\
\hline 10 & 14 & M & Germinoma & 200.6 & 63.0 & 62.7 & 49.2 & 21.9 & 21.5 \\
\hline 11 & 14 & $\mathrm{~F}$ & Germinoma & 216.7 & 67.0 & 60.6 & 55.0 & 17.9 & 9.2 \\
\hline 12 & 12 & $\mathrm{~F}$ & Germinoma & 353.8 & 62.5 & 58.9 & 47.4 & 24.2 & 19.5 \\
\hline
\end{tabular}


of discrete gantry positions per rotation for IMRT planning, and two or more gantry angles that made the minimum average normal brain dose and didn't pass the eyes for 3D-CRT and PBT planning. SOBP for PBT plans were prescribed by a $1 \mathrm{~cm}$ unit and chosed the smallest width as far as PTV covers were enough in the condition. Leaf margin of the PBT plans and 3D-CRT plans also optimized the smallest width as far as PTV covers were enough in the condition. All planning optimization were checked two radiotherapists. Statistical analysis was performed using t-test in SPSS (SPSS Inc., Chicago, IL, USA). Dose-volume histograms (DVHs) for the normal brain were calculated for PBT, IMRT, and 3D-CRT and compared among the methods.

\section{Model cases}

To assess the advantages of PBT based on tumor size and location, model target spheres of different sizes and locations were evaluated. Targets were first prepared with the iso-center at the center of the brain and diameters of 2, 3, 4, and $6 \mathrm{~cm}$ to analyze the effect of target size. Secondly, a target of $4 \mathrm{~cm}$ in diameter was moved from the center of the brain to the peripheral region horizontally in a right-left direction (x-axis) at $1-\mathrm{cm}$ intervals to analyze the effect of target location. Dose distributions for each target were calculated for PBT, 3D-CRT, and IMRT, and the normal brain dose was evaluated as a percentage of the prescription dose. The relative decrease in normal brain dose in PBT compared to 3DCRT or IMRT was calculated from the equation:

The relative decrease in brain average dose

IMRT or 3D - CRT brain average dose - PBT brain average dose IMRT or 3D-CRT brain average dose

\section{Results}

\section{Ependymoma cases (local irradiation)}

Doses to normal brain tissue in PBT, 3D-CRT, and IMRT for the 6 patients with ependymoma are shown in Fig. 1. In the respective methods, the average normal brain doses were $15.5,25.8$, and $26.8 \%$ of the prescription dose. Normal brain doses were significantly lower in PBT compared to 3D-CRT $(p=0.001)$ and IMRT ( $p=$ $0.003)$, with differences ranging from 5.7 to $18.3 \%$ (median 10.5\%). The relative decreases were 28.2 to $63.4 \%$ (median $43.4 \%$ ) compared to 3D-CRT and 28.4 to $62.0 \%$ (median $44.7 \%$ ) compared to IMRT. There was no significant difference in the normal brain dose between IMRT and 3D-CRT $(p=0.296)$.

\section{Germinoma cases (whole-ventricle irradiation)}

Doses to normal brain tissue in PBT, 3D-CRT, and IMRT for the 6 patients with germinoma are shown in

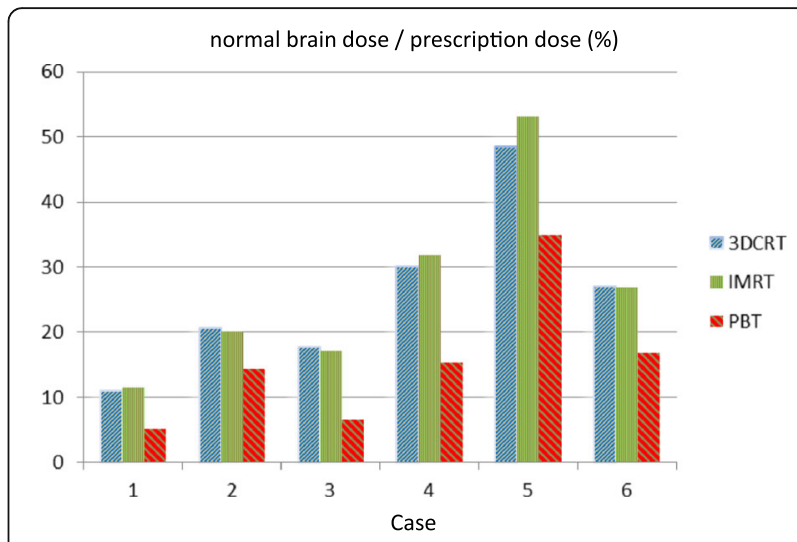

Fig. 1 Average normal brain doses for patients with ependymoma

Fig. 2. The average normal brain doses were 50.6, 63.6, and $62.1 \%$ of the prescription dose, respectively. Normal brain doses were significantly smaller in PBT compared to 3D-CRT and IMRT (both $p=0.000$ ), with differences ranging from 5.6 to $15.1 \%$ (median 12.5\%). The relative decreases were 17.3 to $24.1 \%$ (median $20.9 \%$ ) compared to 3D-CRT and 9.2 to $24.9 \%$ (median 20.5\%) compared to IMRT. There was no significant difference in the normal brain dose between IMRT and 3D-CRT $(p=0.287)$.

Figure 3 shows the example of the treatment planning of ependymoma and germinoma in each methods.

\section{Model cases}

The normal brain doses for targets of diameters 2, 3, 4, and $6 \mathrm{~cm}$ with the iso-center at the brain center were $5.5,8.5,14.5$, and $28 \%$ of the prescription dose in PBT; $11.5,17.5,25.5$, and $41.5 \%$ in 3D-CRT; and 13.4, 18.5, 24.9 , and $39.8 \%$ in IMRT, respectively (Fig. 4). For all targets, the average normal brain dose was smaller in PBT plans compared to 3D-CRT $(p=0.008)$ and IMRT $(p=0.001)$, with no significant difference between 3D-CRT and IMRT $(p=0.886)$. There were significant

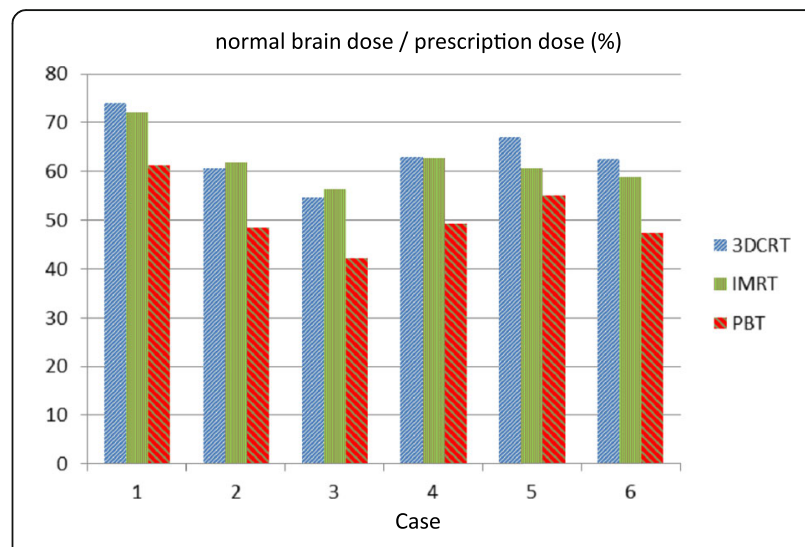

Fig. 2 Average normal brain doses for patients with germinoma 


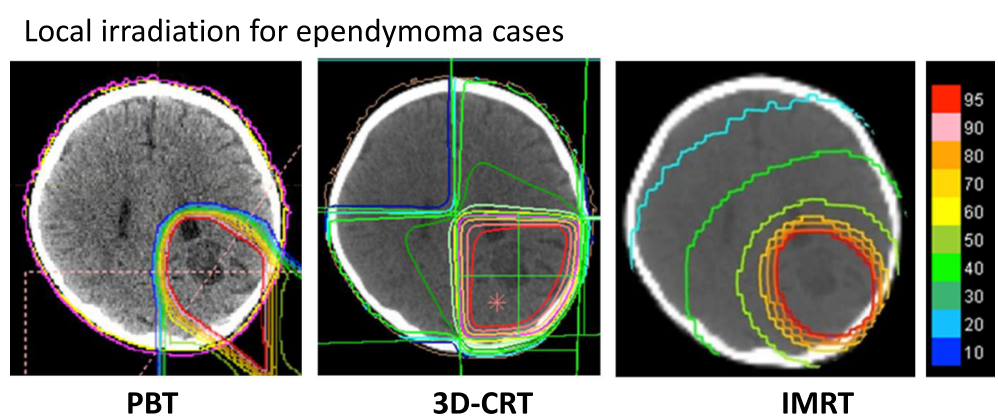

Whole-ventricular irradiation for germinoma cases

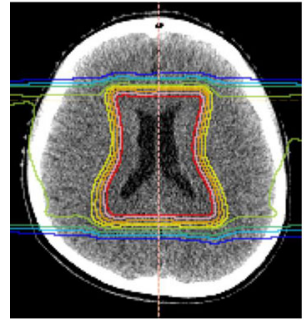

PBT

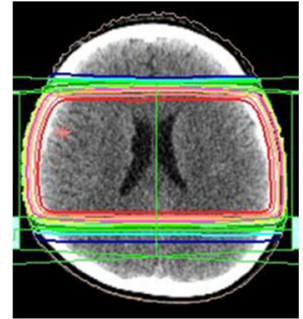

3D-CRT

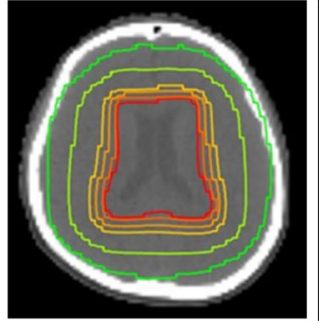

IMRT

Fig. 3 The example of the treatment planning of ependymoma and germinoma in each methods

positive correlations between target size and normal brain dose $(p=0.000$ for PBT, $p=0.002$ for 3D-CRT, $p=0.003$ for IMRT); if the target was larger, the average normal brain dose was larger in all three methods. There were also significant positive correlations between target size and the difference in normal brain dose in PBT compared to 3D-CRT $(p=0.023)$ and IMRT $(p=0.048)$; if the target was larger, there was a larger reduction in normal brain dose in PBT compared to radiotherapy.

\section{a}

(\%) normal brain dose / prescription dose

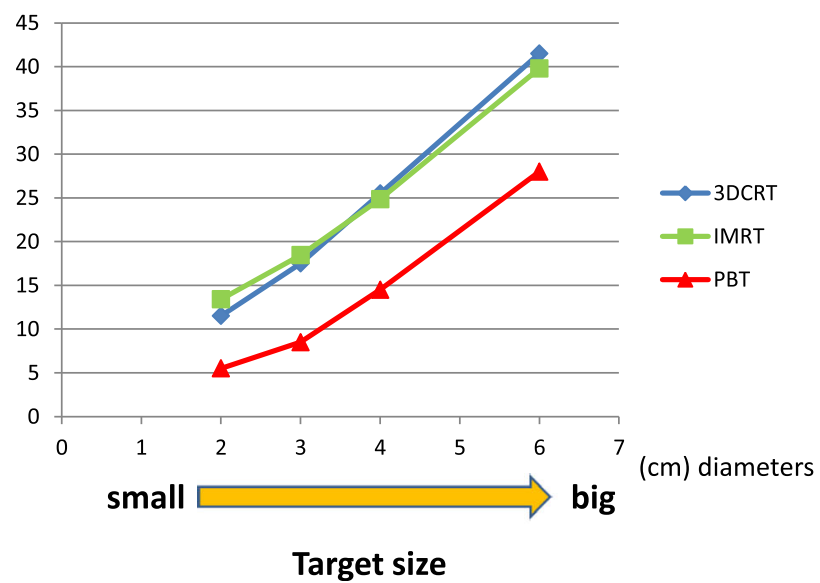

Regarding target location, and with use of bilateral irradiation for 3D-CRT and PBT, the average normal brain doses for targets with iso-centers at $0,1,2,3$, and $4 \mathrm{~cm}$ from the brain center were $14.5 \%, 14.0 \%, 14.0 \%$, $14.0 \%, 14.0 \%$ of the prescription dose for PBT; 25.5, 25.0, 25.0, 25.0, and $24.0 \%$ for 3D-CRT; and 24.9, 24.5, 23.9, $23.0 \%$ and $22.1 \%$ for IMRT, respectively (Fig. 5). For all target locations, the normal brain doses were significantly smaller in PBT plans compared to 3D-CRT and IMRT (both $p=0.000$ ), and in IMRT plans

\section{b}

(\%) difference of the normal brain dose / prescription dose

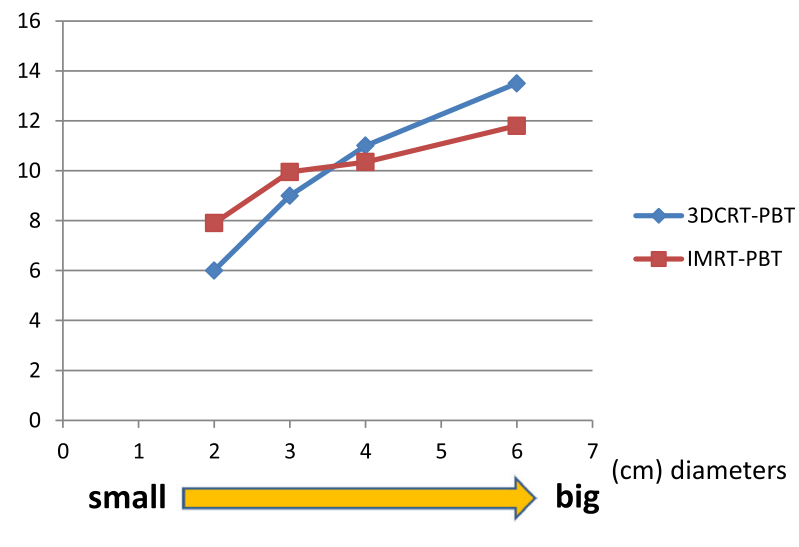

Target size

Fig. 4 a Average normal brain dose for each treatment plan with sphere targets with the same iso-center as the brain center and different diameters $(2,3,4,6 \mathrm{~cm})$. b Correlation between target size and difference in normal brain dose in PBT compared to 3DCRT or IMRT 
average normal brain dose / prescription dose (\%)

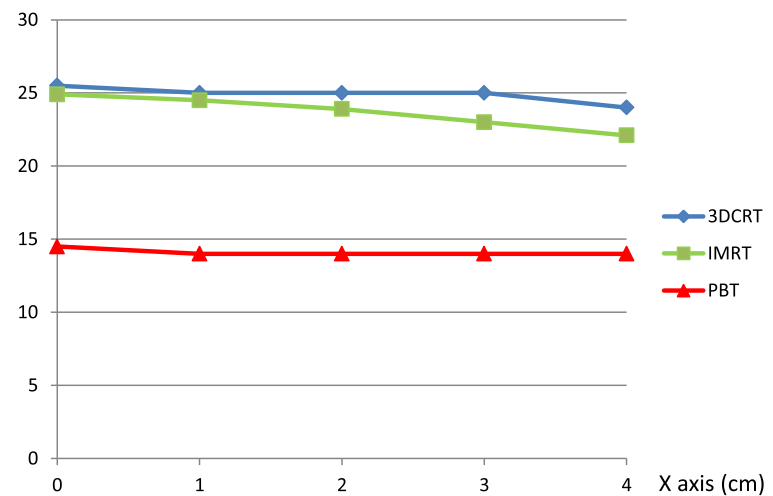

Fig. 5 Average normal brain dose for each treatment plan, using bilateral irradiation for 3D-CRT and PBT, with sphere targets with the same diameter and different iso-centers at $0,1,2,3$, and $4 \mathrm{~cm}$ from the brain center compared to 3D-CRT $(p=0.018)$. There was no correlation between target location and normal brain dose in PBT $(p=0.182)$. In 3D-CRT, the normal brain dose tended to decrease when the target was more peripheral, but the difference was not significant $(p=0.058)$. In IMRT, the normal brain dose was significantly decreased when the target moved peripherally $(p=0.016)$. The difference in normal brain dose between PBT and 3D-CRT was not significantly correlated with target location $(p=0.182)$, but that between PBT and IMRT was significantly correlated with target location $(p=0.014)$.
Using two oblique angle irradiation for 3D-CRT and PBT, the average normal brain doses for targets with iso-centers $0,1,2,3$, and $4 \mathrm{~cm}$ from the brain center were $14.5 \%, 14.0 \%, 12.3 \%, 10.2 \%, 8.4 \%$ of the prescription dose for PBT; and 25.5, 25, 23.5, 22, and $21.5 \%$ for 3D-CRT, respectively (Fig. 6). IMRT gave the same results as those given above. For all target locations, the normal brain dose was significantly smaller in PBT plans compared to 3D-CRT and IMRT (both $p=$ $0.000)$. There was no difference between IMRT and 3D-CRT $(p=0.649)$. There were significant positive correlations between target position and difference in normal brain dose in PBT compared to 3DCRT ( $p=$ $0.006)$ and IMRT $(p=0.004)$; if the target was more peripheral, there was a larger reduction in normal brain dose in PBT compared to radiotherapy.

\section{Discussion}

Proton beam therapy reduces the low dose area for pediatric brain tumor, and it gradually becomes the common recognition [19]. Radiation induce emotional and behavioral deficits and those are remain long time [20]. There were reports indicate that the average dose to the brain is an important factor for future IQ [1]. In this study, we focused on the average dose to normal brain tissue, and we found that PBT can substantially reduce this dose, compared to IMRT and 3D-CRT. This result matches past reports [21,22]. Table 2 shows the difference in the expected IQ after 10 years by using PBT instead of X-ray therapy. PBT was expected decreasing the degree of the 3.4 to 12.8 IQ point in

\section{a}

(\%) normal brain dose / prescription dose

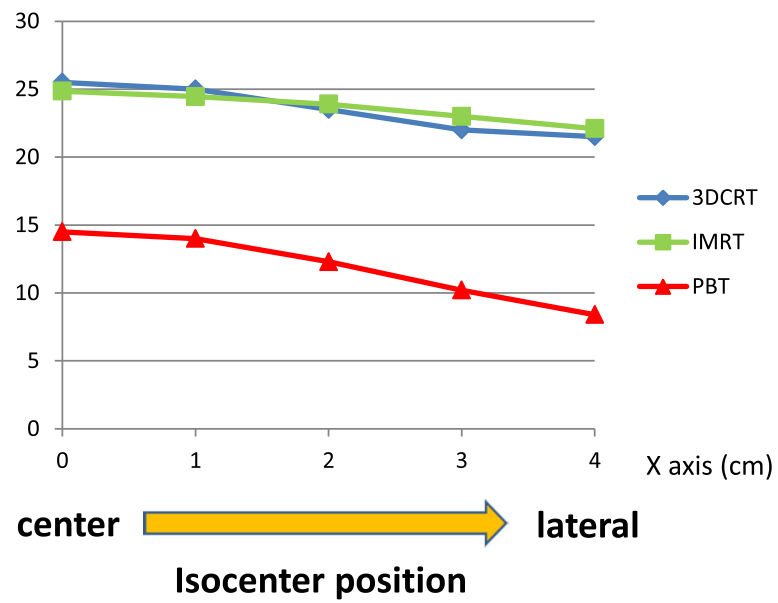

b

(\%) difference of the normal brain dose / prescription dose

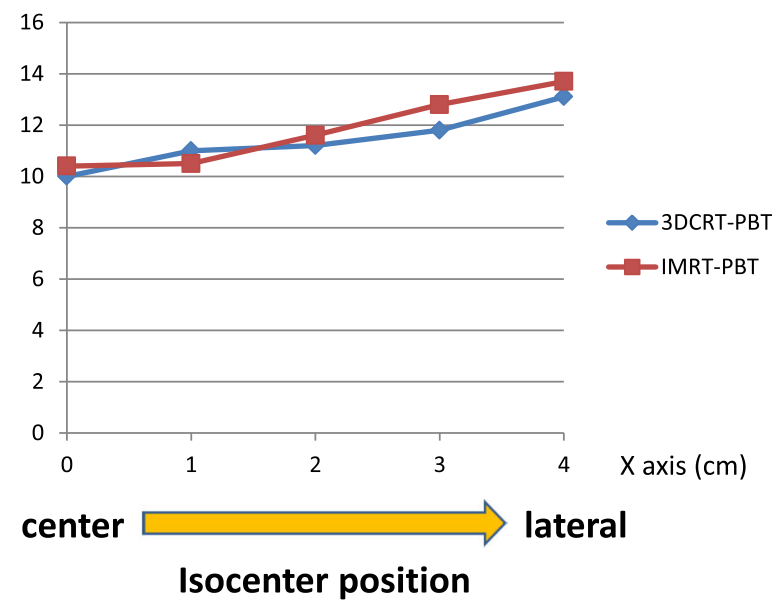

Fig. 6 a Average normal brain dose for each treatment plan, using two oblique angle irradiation for 3D-CRT and PBT, with sphere targets with the same diameter and different iso-centers at $0,1,2,3$, and $4 \mathrm{~cm}$ from the brain center. $\mathbf{b}$ Correlation between target position and difference in normal brain dose in PBT compared to 3DCRT or IMRT 
Table 2 Expected IQ difference after 10 years by using PBT instead of X-ray therapy

\begin{tabular}{|c|c|c|c|c|c|c|c|c|}
\hline No. & Age (y) & Sex & Tumor & $\begin{array}{l}\text { Prescribed } \\
\text { dose (Gy) }\end{array}$ & $\begin{array}{l}\text { Dose difference of the } \\
\text { brain (\%) (3D-CRT-PBT) }\end{array}$ & $\begin{array}{l}\text { Dose difference of the } \\
\text { brain (\%) (IMRT-PBT) }\end{array}$ & $\begin{array}{l}\text { Expected IQ difference } \\
\text { (PBT-3DCRT) }\end{array}$ & $\begin{array}{l}\text { Expected IQ difference } \\
\text { (PBT-IMRT) }\end{array}$ \\
\hline 1. & 6 & M & Ependymoma & 50.4 & 2.97 & 3.23 & 3.4 & 3.7 \\
\hline 2. & 6 & M & Ependymoma & 54.0 & 3.29 & 3.08 & 3.8 & 3.5 \\
\hline 3. & 2 & $\mathrm{~F}$ & Ependymoma & 50.4 & 5.70 & 5.34 & 6.5 & 6.1 \\
\hline 4. & 3 & $\mathrm{~F}$ & Ependymoma & 59.4 & 8.67 & 9.74 & 9.9 & 11.1 \\
\hline 5. & 4 & M & Ependymoma & 61.2 & 8.38 & 11.20 & 9.6 & 12.8 \\
\hline 6. & 3 & $\mathrm{~F}$ & Ependymoma & 45.0 & 4.64 & 4.59 & 5.3 & 5.2 \\
\hline 7. & 11 & M & Germinoma & 30.6 & 3.92 & 3.34 & 4.5 & 3.8 \\
\hline 8. & 16 & $\mathrm{~F}$ & Germinoma & 30.6 & 3.67 & 4.07 & 4.2 & 4.6 \\
\hline 9. & 10 & M & Germinoma & 30.6 & 3.73 & 4.28 & 4.3 & 4.9 \\
\hline 10. & 14 & M & Germinoma & 30.6 & 3.92 & 3.83 & 4.5 & 4.4 \\
\hline 11. & 14 & $\mathrm{~F}$ & Germinoma & 24.0 & 2.88 & 1.34 & 3.3 & 1.5 \\
\hline 12. & 12 & $\mathrm{~F}$ & Germinoma & 30.6 & 4.62 & 3.52 & 5.3 & 4.0 \\
\hline
\end{tabular}

ependymoma cases, and 1.3 to 5.3 point IQ point in germinoma cases. This advantage was found in local irradiation for ependymoma and whole-ventricle irradiation for germinoma, with reductions of almost 40 and $20 \%$, respectively. This effect may produce a 5 -point difference in IQ at entrance to senior high school for a patient with ependymoma who received radiotherapy at 4 years old, and a 3-point difference at high-school graduation for a patient with germinoma who received radiotherapy at age 13.

The advantages of PBT compared to photon radiotherapy including IMRT have been described in treatment of pediatric CNS tumors [9-11]. MacDonald et al. found that proton beams can achieve tumor coverage as well as IMRT, but that normal tissue sparing was better in PBT for patients with germ cell tumor and ependymoma $[17,18]$. Our results are similar to these reports [5, 23]. In addition, PBT is more effective than IMRT or 3D-CRT for treatment of a large or peripheral tumor. For tumor location, the reduction in the dose to normal brain tissue was large when the target was outside over $2 \mathrm{~cm}$ from the brain center. Regarding the model case of the differential target position, we used sphere targets of diameters $4 \mathrm{~cm}$, and this distance was equal to the target radius, which suggests that an advantage of PBT may emerge when the target does not cross the midline of the brain.

From the result of treated case and model case, we found no case that PBT has disadvantage compared with X-ray therapy about normal brain dose, but if the tumor was center and small, the benefit of using PBT was small relatively. Recently it was reported that age and mean radiation dose to specific brain volumes, including the temporal lobes and hippocampi, had a significant impact on longitudinal scores [24]. It is future problem to analyze the risk organ in vain, and it is necessary that assessing not only about brain tumors, but also about body tumors.

\section{Conclusion}

PBT has maximum advantage about normal brain dose for a larger and peripheral tumor, and there was no case that PBT has disadvantage. The dose to normal brain tissue is lower with PBT compared with 3D-CRT and IMRT in local and whole-ventricle irradiation. This information matches past reports.

\section{Acknowledgments}

This research was supported by a grant for Practical Research for Innovative Cancer Control (15ck0106186h0001) from the Japan Agency for Medical Research and Development (AMED). This research was supported in part by Grants-in-Aid for Scientific Research (B) (15H04901) and Young Scientists (B) (25861064) from the Ministry of Education, Science, Sports and Culture of Japan.

\section{Funding}

This work did not receive any specific funding.

Availability of data and materials

The datasets supporting the conclusions of this article are included within the article.

\section{Authors' contributions}

DT made the figure and drafted the manuscript and planned for the treatment cases and model cases, MM planned for treatment cases and drafted the manuscript, YT participated in coordination and revised the article, YO performed the analysis and drafted the manuscript, TF participated in general care and revised the article, $T$ T helped to make all planning, TO participated in coordination and revised the article, KT reviewed and revised the article, HS participated in coordination and helped to draft the manuscript. All authors read and approved the final manuscript.

\section{Competing interests}

The authors declare that they have no competing interests.

\section{Consent for publication}

We obtained consent for publication in written form from the participant or legal parent.

\section{Ethics approval and consent to participate}

All study participants provided informed consent, and we obtained general consent to the research in written form from the participant or legal parent This study was conducted in accordance with the ethical standards defined in the Declaration of Helsinki and was approved by the ethics committee of the University of Tsukuba. 


\section{Author details}

'Department of Radiation Oncology, University of Tsukuba, Tsukuba, Ibaraki, Japan. ${ }^{2}$ Department of Neurosurgery, University of Tsukuba, Tsukuba, Ibaraki, Japan. ${ }^{3}$ Department of Child Health, University of Tsukuba, Tsukuba, Ibaraki, Japan. ${ }^{4}$ Proton Medical Research Center, Graduate School of Comprehensive Human Sciences, University of Tsukuba, Tsukuba, Ibaraki, Japan.

Received: 26 July 2016 Accepted: 6 February 2017

Published online: 22 February 2017

\section{References}

1. Merchant TE, Kiehna EN, Li C, et al. Modeling radiation dosimetry to predict cognitive outcomes in pediatric patients with CNS embryonal tumors includingmedulloblastoma. Int J Radiat Oncol Biol Phys. 2006;65:210-21.

2. Mizumoto M, Okumura T, Hashimoto T, et al. Proton beam therapy for hepatocellular carcinoma: a comparison of three treatment protocols. Int J Radiat Oncol Biol Phys. 2011;81:1039-45.

3. Armoogum KS, Thorp N. Dosimetric comparison and potential for improved clinical outcomes of paediatric CNS patients treated with protons or IMRT. Cancers (Basel). 2015;7:706-22.

4. Fukushima H, Fukushima T, Sakai A, et al. Tailor-made treatment combined with proton beam therapy for children with genitourinary/pelvic rhabdomyosarcoma. Rep Pract Oncol Radiother. 2015;20:217-22.

5. Mizumoto $M$, Oshiro $Y$, Takizawa $D$, et al. Proton beam therapy for pediatric ependymoma. Pediatr Int. 2015;57:567-71.

6. Hoppe-Hirsch E, Brunet L, Laroussinie F, et al. Intellectual outcome in children with malignant tumors of the posterior fossa: influence of the field of irradiation and quality of surgery. Childs Nerv Syst. 1995;11:340-5.

7. Ris MD, Packer R, Goldwein J, Jones-Wallace D, Boyett JM. Intellectual outcome after reduced-dose radiation therapy plus adjuvant chemotherapy for medulloblastoma: a Children's Cancer Group study. J Clin Oncol. 2001;19:3470-6.

8. Spiegler BJ, Bouffet E, Greenberg ML, Rutka JT, Mabbott DJ. Change in neurocognitive functioning after treatment with cranial radiation in childhood. J Clin Oncol. 2004;22:706-13.

9. Walter AW, Mulhern RK, Gajjar A, et al. Survival and neurodevelopmental outcome of young children with medulloblastoma at St Jude Children's Research Hospital. J Clin Oncol. 1999;17:3720-8.

10. Palmer SL, Goloubeva O, Reddick WE, et al. Patterns of intellectual development among survivors of pediatric medulloblastoma: a longitudinal analysis. J Clin Oncol. 2001;19:2302-8.

11. Mostow EN, Byrne J, Connelly RR, Mulvihill JJ. Quality of life in long-term survivors of CNS tumors of childhood and adolescence. J Clin Oncol. 1991;9:592-9.

12. Meadows AT, Gordon J, Massari DJ, Littman P, Fergusson J, Moss K. Declines in IQ scores and cognitive dysfunctions in children with acute lymphocytic leukaemia treated with cranial irradiation. Lancet. 1981;2:1015-8.

13. Kim JY, Park J. Understanding the treatment strategies of intracranial germ cell tumors: focusing on radiotherapy. J Korean Neurosurg Soc. 2015;57(5):315-22.

14. Qi XS, Stinauer M, Rogers B, et al. Potential for improved intelligence quotient using volumetric modulated arc therapy compared with conventional 3-dimensional conformal radiation for whole-ventricular radiation in children. Int J Radiat Oncol Biol Phys. 2012;84(5):1206-11.

15. Taddei PJ, Mirkovic D, Fontenot JD, et al. Stray radiation dose and second cancer risk for a pediatric patient receiving craniospinal irradiation with proton beams. Phys Med Biol. 2009:54:2259-75.

16. Pearce MS, Salotti JA, Little MP, et al. Radiation exposure from CT scans in childhood and subsequent risk of leukaemia and brain tumours: a retrospective cohort study. Lancet. 2012;380:499-505.

17. MacDonald SM, Safai S, Trofimov A, et al. Proton radiotherapy for childhood ependymoma: initial clinical outcomes and dose comparisons. Int J Radiat Oncol Biol Phys. 2008;71:979-86.

18. MacDonald SM, Trofimov A, Safai S, et al. Proton radiotherapy for pediatric central nervous system germ cell tumors: early clinical outcomes. Int $J$ Radiat Oncol Biol Phys. 2011;79:121-9.

19. Yock TI, Yeap BY, Ebb DH, et al. Long-term toxic effects of proton radiotherapy for paediatric medulloblastoma: a phase 2 single-arm study. Lancet Oncol. 2016;17(3):287-98.

20. Willard WW, Conklin HM, Wu S, et al. Prospective longitudinal evaluation of emotional and behavioral functioning in pediatric patients with low-grade glioma treated with conformal radiation therapy. J Neurooncol. 2015; 122(1):161-8.
21. Ares $C$, Albertini $F$, Frei-Welte $M$, et al. Pencil beam scanning proton therapy for pediatric intracranial ependymoma. J Neurooncol. 2016;128(1):137-45.

22. Greenberger BA, Pulsifer MB, Ebb DH, et al. Clinical outcomes and late endocrine, neurocognitive, and visual profiles of proton radiation for pediatric low-grade gliomas. Int J Radiat Oncol Biol Phys. 2014;89(5):1060-8.

23. Mizumoto $M$, Oshiro $Y$, Ayuzawa $K$, et al. Preparation of pediatric patients for treatment with proton beam therapy. Radiother Oncol. 2015;114:245-8.

24. Merchant TE, Schreiber JE, Wu S. Critical combinations of radiation dose and volume predict intelligence quotient and academic achievement scores after craniospinal irradiation in children with medulloblastoma. Int J Radiat Oncol Biol Phys. 2014;90(3):554-61.

\section{Submit your next manuscript to BioMed Central and we will help you at every step:}

- We accept pre-submission inquiries

- Our selector tool helps you to find the most relevant journal

- We provide round the clock customer support

- Convenient online submission

- Thorough peer review

- Inclusion in PubMed and all major indexing services

- Maximum visibility for your research

Submit your manuscript at www.biomedcentral.com/submit
C Biomed Central 